\title{
Influence of Kaolin Sprays on Fruit Quality and Storability of Balady Mandarin
}

\author{
Ali E. Zaghloul ${ }^{1}$, Hassan A. Ennab ${ }^{2}$, Mervat A. El-Shemy ${ }^{1}$
}

\begin{abstract}
The present work was conducted in a private orchard situated at Motobus, Kafr El-Sheikh governorate, Egypt during 2014- 2015 and 2015- 2016 seasons. The main objective of this study is to evaluate the effect of kaolin foliar applications on disorder fruits, yield, fruit quality and storability of Balady mandarin fruits (Citrus reticulate,, $\mathrm{B})$ for 45 days. Trees were sprayed with kaolin at five concentrations $(0,1,2,3$ and $4 \%)$ for three times in summer. Fruit samples were divided into two groups and stored at $2 \pm 1^{\circ} \mathrm{C}$ and $6 \pm 1^{\circ} \mathrm{C}$ at $90-95 \% \mathrm{RH}$ for 45 days.
\end{abstract}

Results showed that, kaolin foliar applications led to increase yield (as number, weight and $(\mathrm{kg})$ of fruits/tree), reduced fruit disorders and improved fruit quality, moreover, it recorded the highest peel fruit firmness, acidity level, soluble solids content (SSC \%) and vitamin C content at harvest time. The highest level of kaolin spray (4\%)significantly enhanced such trials compared to control and the other kaolin concentrations.

In addition, kaolin foliar application at $4 \%$ were the most effective treatment in maintaining fruit quality of Balady mandarin fruits during cold storage at the two storage temperatures $\left(2 \pm 1^{0} \mathrm{C}\right.$ or at $\left.6 \pm 1^{\circ} \mathrm{C}\right)$ for the two seasons, which significantly indicated by reducing weight loss and decay as well as chilling injuries percentages. Also, kaolin treatments at $(4$ and $3 \%)$ recorded the highest values of peel fruit firmness, high levels of SSC, acidity and vitamin $C$ contents compared to the other treatments till the end of storage period, with no significant changes in SSC/acid ratio.

Therefore, spraying with kaolin at $4 \%$ three times in summer months, considered as the most effective treatment in reducing pre-harvest disorder fruits, improving yield and fruit quality at harvest time and maintained good quality and extending storability of Balady mandarin fruits over the other treatments or control during cold storage at $\left(2 \pm 1\right.$ or $\left.6 \pm 1^{\circ} \mathrm{C}\right)$.

Kay words: kaolin, Balady mandarin, Fruit firmness, Weight loss, Disorders, Chilling injury and Citrus reticulate, $\mathrm{B}$

\section{INTRODUCTION}

Citrus productivity as well as fruit quality is affected with a greater extent due to physiological disorders, which resulting from extreme of environmental conditions like temperature, light, and nutritional imbalances (Kumer and Kumer, 2016). In summer,
Balady mandarin fruits exposed to heat stress caused by high temperature, together with direct solar radiation causing fruit damages such as splitting, rind disorders, sunburn and other fruit blemishes (Minessy et al., 1969 and Schrader, 2011). These disorders have a negative impact on yield and fruit quality as well as increase unmarketable fruits and cause high losses to the growers (Verreynne and Merwe, 2011). Furthermore, increasing susceptible fruits to diseases during storage and reduced fruit shelf life. Therefore, kaolin foliar sprays can play an important role in protecting mandarin fruits against sun injuries and physiological disorders (Glen et al., 2002 and Colavita et al., 2011).

Kaolin is a naturally occurring mineral (a clay), main constituent is kaolinite, with the formula $\mathrm{Al}_{4} \mathrm{Si}_{4} \mathrm{O}_{10}(\mathrm{OH})_{8}$ with the following theoretical composition $\mathrm{SiO}_{2}=46.5 \%, \mathrm{Al}_{2} \mathrm{O}_{3}=39.5 \%$ and $\mathrm{H}_{2} \mathrm{O}=$ $14 \%$ (Obaje et al, 2013). (Glenn, 2009) mentioned that, kaolin foliar spray on apple tree to reflect sunlight, led to lower the temperature of fruit surface, reducing sun injuries as well as improving yield and fruit quality. Ennab et al. (2017) concluded that, kaolin foliar applications at 3 and $4 \%$ decreased leaf heat and fruit surface temperature and was more effective to control sunburned fruits of Balady mandarin trees. Moreover, kaolin foliar spray was found to enhance water use efficiency and reducing the adverse effects of water deficit on pistachio and pomegranate trees (Azizi et al., 2013 and El-Khawaga and Mansour(2014). also, kaolin appears to be an important and helpful tool to reduce insect attack, medfly fruit damage and could be a valid alternative to intensive applications of insecticides, currently commonly used in citrus orchards. In this respect, Braham et al. (2007) reported that, Kaolin particle film applications successfully protected fruits from medfly infestations and provided long term control from fruit development until harvest compared to insecticide.

Generally a few studies were done in different fruit species to evaluate the effect of preharvest kaolin treatments on fruit quality during storage on apple (Ergun 2012) and on Washington navel orange( Ali and Elhamahmy 2015).

Therefore, this study aimed to evaluate the effect of kaolin foliar application on physiological disorders,

\footnotetext{
${ }^{1}$ Handling Research Department.

${ }^{2}$ Citrus Research Department

Hort. Res. Inst., Agric. Res. Center (ARC), Giza, Egypt.

Received September 13,2017, Accepted OCTOBR 20, 2017
} 
yield, fruit quality and storability of Balady mandarin fruits.

\section{MATERIALS AND METHODS}

The present study was carried out during 2014-2015 and 2015-2016 seasons on 17 years old Balady mandarin trees (Citrus reticulata, Blanco) budded on Sour orange (Citrus aurantium, L.) rootstock, planted at $5 \times 5$ meter apart in a private orchard situated at Motobus, Kafr El-Sheikh governorate, Egypt. Thirty trees uniform in growth, vigour and productivity were selected and subjected to the same cultural practices commonly adopted on the orchard. Trees were arranged in a randomized complete block design, each treatment replicated three times with two trees per replicate. Kaolin foliar spray was applied as three times, once in 15 June, 15 July and 15 August in both seasons. Treatments included five concentrations of kaolin as follows:

$0,1,2,3$ and $4 \%$.

At harvest time (mid. January in both seasons) when TSS/acid ratio between 10-13, yield of each tree was determined as number and weight $(\mathrm{kg})$ of fruits/tree. The percentages of sunburn, splitting and bruising as fruit disorders was estimated individually by the following general formula:

$$
\text { Plysiological disorders } \%=\frac{\text { Total number of disorder fruits }}{\text { Total number of fruits }} \times 100
$$

A sample of fruits were taken at random from each treatment at harvest time and directly transported to laboratory of Sakha Horticulture Research Station, Kafr El-Sheikh governorate to determine fruit quality. The remaining fruit samples divided into two groups, each group packed in carton, each one contained 28 fruits (replicate). The first group was stored at $2 \pm 1^{\circ} \mathrm{C}$ and 90 $95 \% \mathrm{RH}$ and the second group stored at $6 \pm 1^{\circ} \mathrm{C}$ and 90 $95 \% \mathrm{RH}$ for 45 days. each box represented one replicate. follow:

The variables were measured at 15 days intervals as

Weight loss was expressed as percentage decrease in fruit weight, using the following formula ((W0$\mathrm{Wt} / \mathrm{W} 0)^{*} 100$, with $\mathrm{W} 0$ being the initial weight and $\mathrm{Wt}$ being the fruit weight after an indicated period of storage.

Fruit decay (\%) was determined by counting the number of decayed fruits through 45 days and expressed as a percentage of the initial number after an indicated period of storage as well as chilling injury (\%).Chilling injury symptoms measured as browning area of the peel and internal breakdown. Peel fruit firmness were expressed as $\mathrm{gm} / \mathrm{mm}^{2}$.
Fruit decay (\%) was determined by counting the number of decayed fruits through 45 days and expressed as a percentage of the initial number after an indicated period of storage as well as chilling injury (\%).Chilling injury symptoms measured as browning area of the peel and internal breakdown.

The characteristics quality attributes such as titratable acidity, total soluble solids and ascorbic acid were determined according to A.O.A.C. (1990).

Titratable acidity was measured by titration with 0.1 $\mathrm{N} \mathrm{NaOH}$, and expressed as percentage of citric acid/100 $\mathrm{ml}$ juice. Soluble solids content were measured by a handy refractometer and SSC/acid ratio was estimated. Ascorbic acid was determined by 2,6 dichlorophenol indophenol and expressed as mg ascorbic acid/100 ml juice.

Statistical analysis was done by MSTATC software program and treatment means were compared by using Duncan multiple range test at 5\% level according to Duncan (1955).

\section{RERSULTS AND DISCUSSION}

\section{Effect of kaolin foliar sprays on yield and its components:}

Data in Table (1) showed that, yield as number of fruits per tree, fruit weight and total yield $(\mathrm{kg} / \mathrm{tree})$ of Balady mandarin trees was significantly increased by all kaolin treatments comparing with control in both seasons. The highest yield as ( $\mathrm{kg} / \mathrm{tree})$ was observed with kaolin foliar application of $4 \%$ and $3 \%$ in both seasons, respectively. However, trees sprayed with tap water and kaolin at $1 \%$ gave the lowest yield as number of fruits per tree, fruit weight and yield as ( $\mathrm{kg} / \mathrm{tree})$ compared to other treatments in both seasons. These results are in agreement with those obtained by Ennab et al. (2017) on mandarin. Abdel Ghani et al (2013) on olive concluded that, kaolin foliar spray at $5 \%$ significantly increased yield as number of fruits per tree or weight (kg) of Aggezi and Picual olives compared to foliar spray with calcium carbonate $(5 \%)$ and tap water.

Results in Table (1) also showed that, the heaviest fruits were harvested from trees sprayed with kaolin at $4 \%$ followed by $3 \%$ as compared with those taken from control trees and other treatments, the differences were significant among treatments in both seasons. These results agreed with those of Palitha et al. (2010) on pomegranate, Abd-Allah et al. (2013) on mango and Hegazi et al. (2014) on pomegranate. In this respect, Ennab et al. (2017) using kaolin on mandarin and found that, kaolin treatments at 3 and $4 \%$ improved fruit 
Table 1. Effect of kaolin foliar sprays on yield and its components of Balady mandarin fruits in 2015 and 2016 seasons

\begin{tabular}{lcccccc}
\hline & \multicolumn{5}{c}{ Yield/tree } \\
\cline { 2 - 6 } Treatments & \multicolumn{2}{c}{ Fruits number } & Fruit weight & $\mathbf{( g )}$ & \multicolumn{2}{c}{ Kg/tree } \\
\cline { 2 - 7 } & $\mathbf{2 0 1 5}$ & $\mathbf{2 0 1 6}$ & $\mathbf{2 0 1 5}$ & $\mathbf{2 0 1 6}$ & $\mathbf{2 0 1 5}$ & $\mathbf{2 0 1 6}$ \\
\hline Control & $450.67 \mathrm{~d}$ & $365.33 \mathrm{c}$ & $127.13 \mathrm{~cd}$ & $130.10 \mathrm{~d}$ & $57.29 \mathrm{e}$ & $48.26 \mathrm{e}$ \\
Kaolin 1\% & $476.67 \mathrm{c}$ & $368.67 \mathrm{c}$ & $125.23 \mathrm{~d}$ & $135.52 \mathrm{~d}$ & $59.69 \mathrm{~d}$ & $50.33 \mathrm{~d}$ \\
Kaolin 2\% & $485.33 \mathrm{~b}$ & $380.00 \mathrm{~b}$ & $127.45 \mathrm{~cd}$ & $142.17 \mathrm{c}$ & $61.86 \mathrm{c}$ & $54.02 \mathrm{c}$ \\
Kaolin 3\% & $530.67 \mathrm{a}$ & $391.67 \mathrm{a}$ & $130.81 \mathrm{~b}$ & $148.11 \mathrm{~b}$ & $69.42 \mathrm{~b}$ & $58.60 \mathrm{~b}$ \\
Kaolin 4\% & $532.00 \mathrm{a}$ & $400.33 \mathrm{a}$ & $135.15 \mathrm{a}$ & $152.71 \mathrm{a}$ & $71.69 \mathrm{a}$ & $61.13 \mathrm{a}$ \\
\hline
\end{tabular}

Means followed by different letter are significantly different within columns by Duncan's multiple range test, $\mathrm{P} \leq 0.05$

quality in terms of fruit size, diameter, weight, peel thickness as compared to untreated treatment.

Increased fruit yield as a result of kaolin treatments may be due to its protective effect from high temperature and reflection of solar radiation, especially UV wavelengths, which led to reduce heat stress on fruits, enhance fruit water content by decreasing transpiration from fruit surface (Glenn and Puterka 2005). Also, yield increased as a result of increased fruit number resulting to successfully protected fruits from medfly infestations, reduction fruit disorders and weight.

2.Effect of preharvest kaolin foliar sprays on some fruit disorder percentages of Balady mandarin trees during 2015 and 2016 seasons.

Illustrated data in Table (2) indicated that, all fruit disorder percentages were significantly decreased by kaolin foliar application compared to control in both seasons. Kaolin treatments at 4 and 3\% gave the lowest disorder percentages in both seasons, respectively. Highest percentage was obtained with control treatment in both seasons. However, kaolin foliar application at 1 and $2 \%$ gave intermediate values. These results were in agreement with those obtained by Weerakkody et al. (2010), Vatandoost et al. (2014), Hegazi et al. (2014), Chabbal et al. (2014) and Ennab et al. (2017).

Decreasing fruit disorders resulting kaolin treatments may be due to the protection from high temperature and reflection of solar radiation which led to reduce heat stress on fruits and enhance fruit water content by decreasing transpiration from fruit surface (Glenn and Puterka ,2005). These results are in agreement with those obtained by Glenn et al. (2002) on apple and Colavita et al. (2011) on pear. This reduction in fruit disorders enhances yield and marketable fruit percentage.

\section{Effect of preharvest kaolin foliar sprays on fruit physical characters during cold storage:}

\subsection{Weight loss (\%):}

Data in Table (3) showed that, the percentage of weight loss increased with the progress of storage period and with the higher storage temperature $\left(6 \pm 1^{\circ} \mathrm{C}\right)$ than the lower $\left(2 \pm 1^{0} \mathrm{C}\right)$ during the two seasons. Different kaolin concentrations had lower percentages of weight loss compared to those control. In both storage temperatures, the highest weight loss percentage was observed in control treatment followed by $1 \%$ kaolin treatment. On the other hand, the treatment of $4 \%$ kaolin showed to be the superior one in reducing fruit weight loss percentage during the two seasons, respectively. Whereas, kaolin at concentrations 2 and $3 \%$ gave intermediate values of weight loss.

Generally, the results indicated that, preharvest foliar applications of kaolin was effective in reducing weight loss percentage of Balady mandarin fruits during storage. Similar results were obtained by Du Plooy (2006) on mango. In this respect, Ergun (2012) mentioned that, kaolin film application reduced weight loss ratio during the period of storage compared to the unsprayed ones when used at $2.5 \%$

Table 2. Effect of kaolin foliar sprays some fruit disorders percentages of Balady mandarin fruits in 2015 and 2016 seasons

\begin{tabular}{lcccccccc}
\hline \multirow{2}{*}{ Trestments } & \multicolumn{2}{c}{ Sunburn } & \multicolumn{2}{c}{ Splitting } & \multicolumn{2}{c}{ Bruising } & \multicolumn{2}{c}{ Total } \\
\cline { 2 - 8 } & $\mathbf{2 0 1 5}$ & $\mathbf{2 0 1 6}$ & $\mathbf{2 0 1 5}$ & $\mathbf{2 0 1 6}$ & $\mathbf{2 0 1 5}$ & $\mathbf{2 0 1 6}$ & $\mathbf{2 0 1 5}$ & $\mathbf{2 0 1 6}$ \\
\hline Control & $19.11 \mathrm{a}$ & $15.20 \mathrm{a}$ & $8.16 \mathrm{a}$ & $7.68 \mathrm{a}$ & $6.16 \mathrm{a}$ & $5.48 \mathrm{a}$ & $33.43 \mathrm{a}$ & $28.36 \mathrm{a}$ \\
Kaolin 1 \% & $14.18 \mathrm{~b}$ & $10.73 \mathrm{~b}$ & $6.04 \mathrm{~b}$ & $6.48 \mathrm{~b}$ & $4.17 \mathrm{~b}$ & $4.51 \mathrm{~b}$ & $24.39 \mathrm{~b}$ & $21.72 \mathrm{~b}$ \\
Kaolin 2\% & $10.25 \mathrm{c}$ & $7.33 \mathrm{c}$ & $5.63 \mathrm{c}$ & $5.24 \mathrm{c}$ & $4.22 \mathrm{~b}$ & $3.62 \mathrm{c}$ & $20.10 \mathrm{c}$ & $16.19 \mathrm{c}$ \\
Kaolin 3\% & $6.67 \mathrm{~d}$ & $5.91 \mathrm{~d}$ & $4.35 \mathrm{~d}$ & $3.82 \mathrm{~d}$ & $3.46 \mathrm{c}$ & $2.18 \mathrm{~d}$ & $14.48 \mathrm{~d}$ & $11.91 \mathrm{~d}$ \\
Kaolin 4 \% & $3.15 \mathrm{e}$ & $2.81 \mathrm{e}$ & $2.17 \mathrm{e}$ & $1.58 \mathrm{e}$ & $1.71 \mathrm{~d}$ & $1.46 \mathrm{e}$ & $7.03 \mathrm{e}$ & $5.85 \mathrm{e}$ \\
\hline
\end{tabular}

Means followed by different letter are significantly different within columns by Duncan's multiple range test, $\mathrm{P} \leq 0.05$. 
Table 3. Effect of kaolin foliar sprays on fruit weight loss (\%) of Balady mandarin during cold storage in 2015 and 2016 seasons

\begin{tabular}{|c|c|c|c|c|c|c|c|c|c|c|}
\hline \multirow{3}{*}{ Treatments } & \multicolumn{5}{|c|}{2015 season } & \multicolumn{5}{|c|}{2016 season } \\
\hline & \multicolumn{10}{|c|}{ Storage periods in days at $2 \pm 1^{0} \mathrm{C}$} \\
\hline & $\mathbf{0}$ & 15 & 30 & 45 & Mean & $\mathbf{0}$ & 15 & 30 & 45 & Mean \\
\hline Control & 0.0 & $5.51 \mathrm{a}$ & $7.32 \mathrm{a}$ & $10.44 \mathrm{a}$ & $7.56 \mathrm{a}$ & 0.0 & $4.31 \mathrm{a}$ & $6.22 \mathrm{a}$ & $10.16 \mathrm{a}$ & $6.90 \mathrm{a}$ \\
\hline Kaolin $1 \%$ & 0.0 & $5.36 \mathrm{a}$ & $6.65 \mathrm{~b}$ & $9.76 \mathrm{~b}$ & $7.08 \mathrm{ab}$ & 0.0 & $4.20 \mathrm{a}$ & $5.42 \mathrm{~b}$ & $9.44 \mathrm{~b}$ & $6.35 \mathrm{ab}$ \\
\hline Kaolin $2 \%$ & 0.0 & $4.50 \mathrm{~b}$ & $6.80 \mathrm{~b}$ & $8.27 \mathrm{c}$ & $6.62 \mathrm{abc}$ & 0.0 & $3.29 \mathrm{ab}$ & $5.54 \mathrm{~b}$ & $7.36 \mathrm{c}$ & $5.37 \mathrm{abc}$ \\
\hline Kaolin $3 \%$ & 0.0 & $3.67 \mathrm{c}$ & $5.36 \mathrm{c}$ & $8.24 \mathrm{c}$ & $6.10 \mathrm{bc}$ & 0.0 & $2.54 \mathrm{ab}$ & $4.44 \mathrm{c}$ & $7.33 \mathrm{c}$ & $4.77 \mathrm{bc}$ \\
\hline Kaolin $4 \%$ & 0.0 & $3.33 \mathrm{~d}$ & $4.54 \mathrm{~d}$ & $8.11 \mathrm{c}$ & $5.72 \mathrm{c}$ & 0.0 & $2.15 \mathrm{~b}$ & $3.38 \mathrm{~d}$ & $7.05 \mathrm{~d}$ & $4.19 \mathrm{c}$ \\
\hline \multirow[t]{2}{*}{ Mean } & 0.0 & 4.47 & 6.13 & 8.96 & & 0.0 & 3.29 & 5.00 & 8.26 & \\
\hline & \multicolumn{10}{|c|}{ Storage periods in days at $6 \pm 1^{0} \mathrm{C}$} \\
\hline Control & 0.0 & $7.65 \mathrm{a}$ & $12.95 \mathrm{a}$ & $18.61 \mathrm{a}$ & $12.77 \mathrm{a}$ & 0.0 & $6.30 \mathrm{a}$ & $12.34 \mathrm{a}$ & $18.19 \mathrm{a}$ & $12.27 \mathrm{a}$ \\
\hline Kaolin $1 \%$ & 0.0 & $7.21 \mathrm{~b}$ & $11.92 b$ & $17.68 \mathrm{~b}$ & $12.30 \mathrm{ab}$ & 0.0 & $6.02 \mathrm{~b}$ & $11.85 \mathrm{~b}$ & $17.81 \mathrm{~b}$ & $11.89 \mathrm{a}$ \\
\hline Kaolin $2 \%$ & 0.0 & $6.86 \mathrm{c}$ & $11.34 \mathrm{c}$ & $17.54 b c$ & $11.91 \mathrm{abc}$ & 0.0 & $5.87 \mathrm{~b}$ & $11.16 \mathrm{c}$ & $16.77 \mathrm{c}$ & $11.26 \mathrm{ab}$ \\
\hline Kaolin 3\% & 0.0 & $6.34 \mathrm{~d}$ & $10.51 \mathrm{~d}$ & $17.35 \mathrm{c}$ & $11.50 \mathrm{bc}$ & 0.0 & $5.41 \mathrm{c}$ & $10.16 \mathrm{~d}$ & $16.45 \mathrm{c}$ & $10.67 \mathrm{ab}$ \\
\hline Kaolin $4 \%$ & 0.0 & $5.63 \mathrm{e}$ & $10.14 \mathrm{e}$ & $16.49 \mathrm{~d}$ & $10.99 \mathrm{c}$ & 0.0 & $4.53 \mathrm{~d}$ & $10.12 \mathrm{~d}$ & $15.25 \mathrm{~d}$ & $9.96 \mathrm{~b}$ \\
\hline Mean & 0.0 & 6.73 & 11.37 & 17.53 & & 0.0 & 5.62 & 11.12 & 16.89 & \\
\hline
\end{tabular}

Means followed by different letter are significantly different within columns by Duncan's multiple range test, $\mathrm{P} \leq 0.05$

Table 4. Effect of kaolin foliar sprays on fruit decay (\%) of Balady mandarin during cold storage in 2015 and 2016 seasons

\begin{tabular}{|c|c|c|c|c|c|c|c|c|c|c|}
\hline \multirow{3}{*}{ Treatments } & \multicolumn{5}{|c|}{2015 season } & \multicolumn{5}{|c|}{2016 season } \\
\hline & \multicolumn{10}{|c|}{ Storage periods in days at $2 \pm 1^{0} \mathrm{C}$} \\
\hline & $\mathbf{0}$ & 15 & 30 & 45 & Mean & $\mathbf{0}$ & 15 & 30 & 45 & Mean \\
\hline Control & 0.0 & $4.76 \mathrm{a}$ & $7.14 \mathrm{a}$ & $14.28 \mathrm{a}$ & $8.73 \mathrm{a}$ & 0.0 & $4.76 \mathrm{a}$ & $7.14 \mathrm{a}$ & $9.52 \mathrm{a}$ & $7.14 \mathrm{a}$ \\
\hline Kaolin $1 \%$ & 0.0 & $4.76 \mathrm{a}$ & $7.14 \mathrm{a}$ & $11.90 \mathrm{~b}$ & $7.93 \mathrm{ab}$ & 0.0 & $4.76 \mathrm{a}$ & $7.14 \mathrm{a}$ & $9.52 \mathrm{a}$ & $7.14 \mathrm{a}$ \\
\hline Kaolin $2 \%$ & 0.0 & $4.76 \mathrm{a}$ & $7.14 \mathrm{a}$ & $11.90 \mathrm{~b}$ & $7.93 \mathrm{ab}$ & 0.0 & $2.38 \mathrm{~b}$ & $4.76 \mathrm{~b}$ & $9.52 \mathrm{a}$ & $5.55 \mathrm{~b}$ \\
\hline Kaolin 3\% & 0.0 & $2.38 \mathrm{~b}$ & $7.14 \mathrm{a}$ & $11.90 \mathrm{~b}$ & $7.14 \mathrm{bc}$ & 0.0 & $0.00 \mathrm{c}$ & $2.38 \mathrm{c}$ & $7.14 \mathrm{~b}$ & $3.17 \mathrm{c}$ \\
\hline Kaolin $4 \%$ & 0.0 & 0.00 & $4.76 \mathrm{~b}$ & $9.52 \mathrm{c}$ & $4.76 \mathrm{c}$ & 0.0 & $0.00 \mathrm{c}$ & $2.38 \mathrm{c}$ & $7.14 \mathrm{~b}$ & $3.17 \mathrm{c}$ \\
\hline \multirow[t]{2}{*}{ Mean } & 0.0 & 3.33 & 6.66 & 11.90 & & 0.00 & 2.38 & 4.76 & 9.04 & \\
\hline & \multicolumn{9}{|c|}{ Storage periods in days at $6 \pm 1^{0} \mathrm{C}$} & \\
\hline Control & 0.0 & $7.14 \mathrm{a}$ & $14.28 \mathrm{a}$ & $19.05 \mathrm{a}$ & $11.90 \mathrm{a}$ & 0.0 & $7.14 \mathrm{a}$ & $11.90 \mathrm{a}$ & $16.67 \mathrm{a}$ & $11.90 \mathrm{a}$ \\
\hline Kaolin $1 \%$ & 0.0 & $7.14 \mathrm{a}$ & $11.90 \mathrm{~b}$ & 19.05 & $9.53 \mathrm{ab}$ & 0.0 & $7.14 \mathrm{a}$ & $11.90 \mathrm{a}$ & $14.29 \mathrm{~b}$ & $11.11 \mathrm{ab}$ \\
\hline Kaolin $2 \%$ & 0.0 & $7.14 \mathrm{a}$ & $11.90 \mathrm{~b}$ & $16.67 \mathrm{~b}$ & $9.50 \mathrm{ab}$ & 0.0 & $4.76 \mathrm{~b}$ & $11.90 \mathrm{a}$ & $14.29 \mathrm{~b}$ & $10.32 \mathrm{ab}$ \\
\hline Kaolin 3\% & 0.0 & $4.76 \mathrm{~b}$ & $11.90 \mathrm{~b}$ & $16.67 \mathrm{~b}$ & $7.61 \mathrm{~b}$ & 0.0 & $4.76 \mathrm{~b}$ & $9.52 \mathrm{~b}$ & $14.29 \mathrm{~b}$ & $9.52 b c$ \\
\hline Kaolin $4 \%$ & 0.0 & $4.76 b$ & $9.52 \mathrm{c}$ & $14.29 \mathrm{c}$ & $7.14 \mathrm{~b}$ & 0.0 & $4.76 \mathrm{~b}$ & $7.14 \mathrm{c}$ & $11.90 \mathrm{c}$ & $7.93 \mathrm{c}$ \\
\hline Mean & 0.0 & 6.19 & 11.90 & 17.15 & & 0.00 & 5.71 & 10.47 & 14.29 & \\
\hline
\end{tabular}

Means followed by different letter are significantly different within columns by Duncan's multiple range test, $\mathrm{P} \leq .05$.

and sprayed three time before harvest on Galaxy apple trees stored at $6^{0} \mathrm{C}$ for 100 days. The loss of water from fresh fruit after harvest is a serious problem, causing shrinkage and weight loss. The reduction in fruit weight loss as a result of foliar application of kaolin maybe due to reduce dehydration and water loss, retain the shriveling of the fruit skin, delay the fruit ripening and thereby delay the decline in fruit quality.

\subsection{Decay (\%):}

Decay percentages of kaolin sprayed fruits was lower compared to the unsprayed ones at the end of storage period at two different storage temperatures $\left(2 \pm 1^{\circ} \mathrm{C}\right.$ and $\left.6 \pm 1^{0} \mathrm{C}\right)$ Table (4).

Storage at $2^{0} \mathrm{C}$ showed lower decay percentage than that at $6^{\circ} \mathrm{C}$. Decayed fruits were increased with the prolonging of storage period and high storage temperature. The highest decay\% was observed in control (water sprayed trees) treatment followed by $1 \%$ and $2 \%$ kaolin in both seasons, respectively. Whereas, kaolin foliar application at 4 and $3 \%$ was more effective in reducing fruit decay percentage compared to other treatments.

One of the main effects of kaolin particle film during fruit postharvest life is to maintain their flesh 
firmness and delay the ripening processes. Similar results were obtained by Aquino et al. (2011) on Satsuma mandarin, grapefruit and orange. Also, Ali and Elhamahmy (2015) reported that, kaolin foliar applications at 2.5 and $5 \%$ were effective in reducing fruit decay $\%$ of Washington navel orange compared to control.

\subsection{Peel fruit firmmess $\left(\mathrm{gm} / \mathrm{mm}^{2)}\right.$ :}

Data in Table (5) cleared that, all kaolin foliar applications showed a positive effect on improving peel fruit firmness of Balady mandarin fruits at harvest time and cold storage in the two seasons, especially at high concentrations (3 and 4\%) compared to control and other treatments on the two storage temperatures during cold storage period in both seasons. This trend was noticed in all measurements till the end of storage time. One of the main effects of kaolin particle film during fruit postharvest life is to maintain their flesh firmness and delay the ripening processes. The highest peel fruit firmness at the end of storage period was recorded with kaolin treatment at $4 \%$ followed by $3 \%$ however, the lowest values were noticed in control treatment during the two seasons and with both storage temperatures during storage period with a marked significant between all treatments and control . This findings are in harmony with that of Ali and Elhamahmy (2015), who reported that, Washington navel orange fruits treated by kaolin particle film at $2.5 \%$ recorded the highest values of fruit firmness compared to control and other treatments. Also, Argiriou and Nanos (2010) reported that, kaolin applications at $4 \%$ had a positive effect on flesh firmness and overall fruit eating quality in Royal Glory

peaches at harvest and during storage. during the two seasons.
Table 5. Effect of kaolin foliar sprays on peel fruit firmness $\left(\mathbf{g m} / \mathbf{m m}^{\mathbf{2}}\right)$ of Balad storage in 2015 and 2016 seasons

\begin{tabular}{|c|c|c|c|c|c|c|c|c|c|c|}
\hline \multirow{3}{*}{ Treatments } & \multicolumn{6}{|c|}{2015 season } & \multicolumn{4}{|c|}{2016 season } \\
\hline & \multicolumn{10}{|c|}{ Storage periods in days at $2 \pm 1^{0} \mathrm{C}$} \\
\hline & $\mathbf{0}$ & 15 & 30 & 45 & Mean & $\mathbf{0}$ & 15 & 30 & 45 & Mean \\
\hline Control & $32.89 \mathrm{e}$ & $31.78 \mathrm{e}$ & $30.73 \mathrm{e}$ & $29.83 \mathrm{e}$ & $31.31 \mathrm{~d}$ & $33.78 \mathrm{e}$ & $32.94 \mathrm{e}$ & $31.51 \mathrm{e}$ & $30.53 \mathrm{e}$ & $32.19 \mathrm{e}$ \\
\hline Kaolin $1 \%$ & $34.97 \mathrm{~d}$ & $33.63 \mathrm{~d}$ & $32.53 \mathrm{~d}$ & $31.29 \mathrm{~d}$ & $33.11 \mathrm{~d}$ & $37.97 \mathrm{~d}$ & $37.83 \mathrm{~d}$ & $36.26 \mathrm{~d}$ & $34.51 \mathrm{~d}$ & $36.64 d$ \\
\hline Kaolin $2 \%$ & $38.33 \mathrm{c}$ & $37.24 \mathrm{c}$ & $35.81 \mathrm{c}$ & $33.77 \mathrm{c}$ & $36.28 \mathrm{c}$ & $44.77 \mathrm{c}$ & $43.25 \mathrm{c}$ & $42.38 \mathrm{c}$ & $41.36 \mathrm{c}$ & $43.05 \mathrm{c}$ \\
\hline Kaolin $3 \%$ & $46.69 \mathrm{~b}$ & $45.39 b$ & $44.47 \mathrm{~b}$ & $43.42 \mathrm{~b}$ & $44.99 \mathrm{~b}$ & $48.13 \mathrm{~b}$ & $46.65 \mathrm{~b}$ & $44.86 \mathrm{~b}$ & $44.10 \mathrm{~b}$ & $44.91 b$ \\
\hline Kaolin $4 \%$ & $54.13 \mathrm{a}$ & $52.68 \mathrm{a}$ & $50.72 \mathrm{a}$ & $48.01 \mathrm{a}$ & $51.39 \mathrm{a}$ & $55.33 \mathrm{a}$ & $54.58 \mathrm{a}$ & $51.75 \mathrm{a}$ & $51.43 \mathrm{a}$ & $53.27 \mathrm{a}$ \\
\hline \multirow[t]{2}{*}{ Mean } & 41.40 & 40.08 & 38.85 & 37.26 & & 43.99 & 43.05 & 41.35 & 40.38 & \\
\hline & \multicolumn{10}{|c|}{ Storage periods in days at $6 \pm 1^{0} \mathrm{C}$} \\
\hline Control & $32.89 \mathrm{e}$ & $30.47 \mathrm{e}$ & $28.37 \mathrm{~d}$ & $24.40 \mathrm{~d}$ & $29.05 \mathrm{e}$ & $33.78 \mathrm{e}$ & $32.28 \mathrm{~d}$ & $29.67 \mathrm{e}$ & $25.79 \mathrm{e}$ & $30.38 \mathrm{e}$ \\
\hline Kaolin $1 \%$ & $34.97 \mathrm{~d}$ & $33.03 \mathrm{~d}$ & $31.43 \mathrm{c}$ & $28.75 \mathrm{c}$ & $32.89 \mathrm{~d}$ & $37.97 \mathrm{~d}$ & $37.13 \mathrm{c}$ & $34.23 \mathrm{~d}$ & $30.41 \mathrm{~d}$ & $34.89 \mathrm{~d}$ \\
\hline Kaolin $2 \%$ & $38.33 \mathrm{c}$ & $36.05 \mathrm{c}$ & $33.55 \mathrm{c}$ & $30.40 \mathrm{c}$ & $34.58 \mathrm{c}$ & $44.77 \mathrm{c}$ & $41.91 b$ & $39.99 \mathrm{c}$ & $34.57 \mathrm{c}$ & $40.31 \mathrm{c}$ \\
\hline Kaolin $3 \%$ & $46.69 \mathrm{~b}$ & $44.02 \mathrm{~b}$ & $40.78 \mathrm{~b}$ & $35.29 \mathrm{~b}$ & $41.70 \mathrm{~b}$ & $48.13 \mathrm{~b}$ & $44.91 \mathrm{~b}$ & $43.01 \mathrm{~b}$ & $38.17 \mathrm{~b}$ & $43.56 b$ \\
\hline Kaolin $4 \%$ & $54.13 \mathrm{a}$ & $51.59 \mathrm{a}$ & $49.22 \mathrm{a}$ & $43.31 \mathrm{a}$ & $49.56 \mathrm{a}$ & $55.33 \mathrm{a}$ & $52.83 \mathrm{a}$ & $51.94 \mathrm{a}$ & $46.52 \mathrm{a}$ & $51.66 \mathrm{a}$ \\
\hline Mean & 41.40 & 39.03 & 36.67 & 32.43 & & 43.99 & 41.81 & 39.76 & 35.09 & \\
\hline
\end{tabular}

Means followed by different letter are significantly different within columns by Duncan's multiple range test, $\mathrm{P} \leq \leq .05$.

\subsection{Chilling injury $(\%)$ :}

In this study data in Table (6) showed that, the first chilling injury symptoms were appeared in control fruit after 15 days of storage at $2^{0} \mathrm{C}$ and after 30 days of storage at $6 \pm 1^{0} \mathrm{C}$ with appearance of featheriness and browning. Moreover, chilling injury symptoms showed an increase with the low kaolin concentrations and control, also with the low storage temperature during cold storage period. Chilling injury symptoms were increased slightly with the progress of storage time. The highest concentration treatments of kaolin (3 and 4\%) did not show any fruit chilling injury symptoms during cold storage at $6 \pm 1{ }^{0} \mathrm{C}$ in the two seasons. Moreover, (4\%) kaolin spray did not recorded any fruit chilling injuries on both storage temperatures till (45 days) of storage in both seasons. Control fruits were significantly reached the highest chilling injury values compared with all kaolin treatments at the two storage temperatures at the two seasons. The above results are in a harmony with that of Ahmed et al. (2013) and Mditshwa et al. (2013). Thes concluded that, silicon dips have an ability to reduce chilling injury symptoms in lemons.

\subsection{Effect of preharvest kaolin foliar sprays on fruit chemical characters during cold storage}

\subsection{Soluble solids content (SSC) \% :}

Soluble solids content (SSC) in Balady mandarin fruits decreased gradually as storage time progressed and with the highest storage temperature, Table (7).

The values of SSC of control at both storage temperatures were lower than those of kaolin treatments during the two seasons. 
Table 6. Effect of kaolin foliar sprays on fruit chilling injury \% of Balady mandarin during cold storage in 2015 and 2016 seasons

\begin{tabular}{|c|c|c|c|c|c|c|c|c|c|c|}
\hline \multirow{3}{*}{ Treatments } & \multicolumn{5}{|c|}{2015 season } & \multicolumn{5}{|c|}{2016 season } \\
\hline & \multicolumn{10}{|c|}{ Storage periods in days at $2 \pm 1^{0} \mathrm{C}$} \\
\hline & $\mathbf{0}$ & 15 & 30 & 45 & Mean & $\mathbf{0}$ & 15 & 30 & 45 & Mean \\
\hline Control & 0.00 & $1.19 \mathrm{a}$ & $2.38 \mathrm{a}$ & $4.76 \mathrm{a}$ & $2.08 \mathrm{a}$ & 0.00 & $1.19 \mathrm{a}$ & $2.38 \mathrm{a}$ & $4.76 \mathrm{a}$ & $2.08 \mathrm{a}$ \\
\hline Kaolin $1 \%$ & 0.00 & $1.19 \mathrm{a}$ & $2.38 \mathrm{a}$ & $4.76 \mathrm{a}$ & $2.08 \mathrm{a}$ & 0.00 & $0.00 \mathrm{~b}$ & $2.38 \mathrm{a}$ & $4.76 \mathrm{a}$ & $1.79 \mathrm{~b}$ \\
\hline Kaolin $2 \%$ & 0.00 & $0.00 \mathrm{~b}$ & $1.19 \mathrm{~b}$ & $3.57 \mathrm{~b}$ & $1.19 \mathrm{~b}$ & 0.00 & $0.00 \mathrm{~b}$ & $1.19 \mathrm{~b}$ & $3.57 \mathrm{~b}$ & $1.19 \mathrm{c}$ \\
\hline Kaolin $3 \%$ & 0.00 & $0.00 \mathrm{~b}$ & $1.19 \mathrm{~b}$ & $3.57 \mathrm{~b}$ & $1.19 \mathrm{~b}$ & 0.00 & $0.00 \mathrm{~b}$ & $1.19 \mathrm{~b}$ & $2.38 \mathrm{c}$ & $0.89 \mathrm{~d}$ \\
\hline Kaolin $4 \%$ & 0.00 & $0.00 \mathrm{~b}$ & $0.00 \mathrm{c}$ & $0.00 \mathrm{c}$ & $0.00 \mathrm{c}$ & 0.00 & $0.00 \mathrm{~b}$ & $0.00 \mathrm{c}$ & $0.00 \mathrm{~d}$ & $0.00 \mathrm{e}$ \\
\hline \multirow[t]{2}{*}{ Mean } & 0.00 & 0.48 & 1.43 & 3.33 & 1.31 & 0.00 & 0.24 & 1.43 & 3.09 & 1.19 \\
\hline & \multicolumn{10}{|c|}{ Storage periods in days at $6 \pm 1^{0} \mathrm{C}$} \\
\hline Control & 0.00 & 0.00 & $1.19 \mathrm{a}$ & $2.38 \mathrm{a}$ & $0.89 \mathrm{a}$ & 0.00 & 0.00 & $1.19 \mathrm{a}$ & $2.38 \mathrm{a}$ & $0.84 \mathrm{a}$ \\
\hline Kaolin $1 \%$ & 0.00 & 0.00 & $1.19 \mathrm{a}$ & $2.38 \mathrm{a}$ & $0.89 \mathrm{a}$ & 0.00 & 0.00 & $1.19 \mathrm{a}$ & $1.19 \mathrm{~b}$ & $0.60 \mathrm{~b}$ \\
\hline Kaolin $2 \%$ & 0.00 & 0.00 & $0.00 \mathrm{~b}$ & $1.19 \mathrm{~b}$ & $0.29 \mathrm{~b}$ & 0.00 & 0.00 & $0.00 \mathrm{~b}$ & $1.19 \mathrm{~b}$ & $0.30 \mathrm{c}$ \\
\hline Kaolin $3 \%$ & 0.00 & 0.00 & $0.00 \mathrm{~b}$ & $0.00 \mathrm{c}$ & $0.00 \mathrm{c}$ & 0.00 & 0.00 & $0.00 \mathrm{~b}$ & $0.00 \mathrm{c}$ & $0.00 \mathrm{~d}$ \\
\hline Kaolin $4 \%$ & 0.00 & 0.00 & $0.00 \mathrm{~b}$ & $0.00 \mathrm{c}$ & $0.00 \mathrm{c}$ & 0.00 & 0.00 & $0.00 \mathrm{~b}$ & $0.00 \mathrm{c}$ & $0.00 \mathrm{~d}$ \\
\hline Mean & 0.00 & 0.00 & 0.48 & 1.19 & 0.41 & 0.00 & 0.00 & 0.48 & 0.95 & 0.36 \\
\hline
\end{tabular}

Means followed by different letter are significantly different within columns by Duncan's multiple range test, $\mathrm{P} \leq .05$.

Table 7. Effect of kaolin foliar sprays on fruit SSC (\%) of Balady mandarin during storage cold in 2015 and 2016 seasons

\begin{tabular}{|c|c|c|c|c|c|c|c|c|c|c|}
\hline \multirow{3}{*}{ Treatments } & \multicolumn{5}{|c|}{2015 season } & \multicolumn{5}{|c|}{2016 season } \\
\hline & \multicolumn{10}{|c|}{ Storage periods in days at $2 \pm 1^{0} \mathrm{C}$} \\
\hline & $\mathbf{0}$ & 15 & 30 & 45 & Mean & $\mathbf{0}$ & 15 & 30 & 45 & Mean \\
\hline Control & $11.28 \mathrm{e}$ & $11.13 \mathrm{c}$ & $11.06 \mathrm{c}$ & $10.86 \mathrm{c}$ & $11.08 \mathrm{~d}$ & $11.51 \mathrm{~d}$ & $11.33 \mathrm{c}$ & $11.13 \mathrm{c}$ & $10.93 \mathrm{e}$ & $11.23 \mathrm{e}$ \\
\hline Kaolin $1 \%$ & $11.79 \mathrm{~d}$ & $11.80 \mathrm{~b}$ & $11.66 \mathrm{~b}$ & $11.53 \mathrm{~b}$ & $11.70 \mathrm{c}$ & $12.15 \mathrm{c}$ & $12.00 \mathrm{~b}$ & $11.86 \mathrm{~b}$ & $11.60 \mathrm{~d}$ & $11.94 \mathrm{~d}$ \\
\hline Kaolin $2 \%$ & $12.33 \mathrm{c}$ & $11.93 \mathrm{ab}$ & $11.93 \mathrm{a}$ & $11.80 \mathrm{a}$ & $11.99 \mathrm{~b}$ & $12.31 \mathrm{~b}$ & $12.00 \mathrm{~b}$ & $12.00 \mathrm{ab}$ & $11.80 \mathrm{c}$ & $12.08 \mathrm{c}$ \\
\hline Kaolin 3\% & $12.41 \mathrm{~b}$ & $12.00 \mathrm{ab}$ & $11.93 \mathrm{a}$ & $11.86 \mathrm{a}$ & $12.05 \mathrm{ab}$ & $12.50 \mathrm{a}$ & $12.26 \mathrm{ab}$ & $12.13 \mathrm{ab}$ & $11.93 \mathrm{~b}$ & $12.21 \mathrm{~b}$ \\
\hline Kaolin4 \% & $12.51 \mathrm{a}$ & $12.13 \mathrm{a}$ & $12.00 \mathrm{a}$ & $11.95 \mathrm{a}$ & $12.14 \mathrm{a}$ & $12.56 \mathrm{a}$ & $12.43 \mathrm{a}$ & $12.26 \mathrm{a}$ & $12.13 \mathrm{a}$ & $12.35 \mathrm{a}$ \\
\hline \multirow[t]{2}{*}{ Mean } & 12.06 & 11.79 & 11.71 & 11.59 & & 12.20 & 12.00 & 11.87 & 11.67 & \\
\hline & \multicolumn{10}{|c|}{ Storage periods in days at $6 \pm 1^{0} \mathrm{C}$} \\
\hline Control & $11.28 \mathrm{e}$ & $10.80 \mathrm{~d}$ & $10.60 \mathrm{c}$ & $10.20 \mathrm{c}$ & $10.72 \mathrm{c}$ & $11.51 \mathrm{~d}$ & $11.26 \mathrm{c}$ & $11.06 \mathrm{c}$ & $10.73 \mathrm{~d}$ & $11.14 \mathrm{~d}$ \\
\hline Kaolin $1 \%$ & $11.79 \mathrm{~d}$ & $11.66 \mathrm{c}$ & $11.46 \mathrm{~b}$ & $11.13 \mathrm{~b}$ & $11.51 \mathrm{~b}$ & $12.15 \mathrm{c}$ & $11.86 \mathrm{~b}$ & $11.73 \mathrm{~b}$ & $11.33 \mathrm{c}$ & $11.77 \mathrm{c}$ \\
\hline Kaolin $2 \%$ & $12.3 \mathrm{c}$ & $12.06 \mathrm{ab}$ & $11.86 \mathrm{a}$ & $11.46 \mathrm{a}$ & $11.93 \mathrm{a}$ & $12.31 \mathrm{~b}$ & $12.06 \mathrm{ab}$ & $12.00 \mathrm{a}$ & $11.56 \mathrm{~b}$ & $11.98 b c$ \\
\hline Kaolin 3\% & $12.41 \mathrm{~b}$ & $11.86 \mathrm{bc}$ & $11.80 \mathrm{a}$ & $11.46 \mathrm{a}$ & $11.88 \mathrm{ab}$ & $12.50 \mathrm{a}$ & $12.13 \mathrm{ab}$ & $12.06 \mathrm{a}$ & $11.66 \mathrm{~b}$ & $12.09 \mathrm{ab}$ \\
\hline Kaolin4 \% & $12.51 \mathrm{a}$ & $12.20 \mathrm{a}$ & $11.93 \mathrm{a}$ & $11.60 \mathrm{a}$ & $12.06 \mathrm{a}$ & $12.56 \mathrm{a}$ & $12.30 \mathrm{a}$ & $12.13 \mathrm{a}$ & $11.86 \mathrm{a}$ & $12.21 \mathrm{a}$ \\
\hline Mean & 12.06 & 11.71 & 11.53 & 11.17 & & 12.20 & 11.92 & 11.79 & 11.00 & \\
\hline
\end{tabular}

Means followed by different letter are significantly different within columns by Duncan's multiple range test, $\mathrm{P} \leq 0.05$.

High concentrations of Kaolin (4 and 3\%) improved fruit SSC \% at harvest time and maintained high level of it at different period of cold storage till the end of storage time compared to control and other treatments. At the end of the storage period, the highest SSC values were recorded in kaolin foliar sprayed fruits at $4 \%$ followed by $3 \%$, respectively, while the lowest SSC was found in control treatments in both seasons and at both stored temperatures. Similar findings were observed in some fruits treated with kaolin, as those reported by Argiriou and Nanos (2010) on peaches, Schrader et al. (2009) on apple and Aquino et al. (2011) on citrus and stone fruits. The increase in total soluble solids in fruits as a result of kaolin sprays may be related to the increased activity of enzymes responsible for the hydrolysis of starch to soluble sugars and the conversion of starch to sugar, which indicated that, the fruits are in the ripening process. The increase in total soluble solids in fruits as a result of kaolin sprays may be related to its effect on delaying ripening process, senescence stage and loss postharvest fruit quality as SSC. This explanation agreed with those obtained by (Ali and Elhamahmy 2015) on Washington navel orange fruits.

\section{2. Titratable acidity $(\%)$ :}

Data presented in Table (8) showed that, all kaolin concentrations recorded high levels of titratable acidity in mandarin fruits during storage period compared to 
unsprayed fruits. In addition titratable acidity values were gradually decreased with increasing storage period at the two storage temperatures. On the other words, the highest storage temperature increased the decreament of TA with all treatments during storage period. The highest values of titratable acidity were observed in the $4 \%$ kaolin sprayed fruits with a high significant differences compared with the other treatments in most cases. These results were similar with those reported by Argiriou and Nanos (2010) on peaches and Ergun (2012) on apple. In this respect, Ali and Elhamahmy (2015) reported that, Washington navel orange fruits sprayed with kaolin at $2.5 \%$ recorded maximum level of titratable acidity at 100 days from start of storage period. The major organic acids in citrus fruits are citric, ascorbic, oxalic, tartaric, malic, lactic, whereas, citric acid account the most abundant acid of the total acid constituents of the juice. As the ripening of the fruits develops, a reduction in titratable acidity was observed. The decrease in acid content may be due to the use of acids by the fruit as a source of energy and the conversion of organic acids to form sugar (Burton, 1985).

\section{3. SSC/acid ratio:}

The results revealed that, the SSC/acid ratio increased as the storage period progressed (Table 9). Fruits stored at $2 \pm 1{ }^{\circ} \mathrm{C}$ had slight high values of SSC/acid ratio than that stored at $6 \pm 1{ }^{0} \mathrm{C}$. The differences were not significant among all treatments in most cases at different storage temperatures in both seasons. Similar results were reported by Argiriou and Nanos (2010).

\section{4. Vitamin $C$ content ( $\mathrm{mg} / 100 \mathrm{ml}$ juice):}

Data presented in Table (10) cleared that, higher concentrations of kaolin foliar sprays produced the

Table 8. Effect of kaolin foliar sprays on fruit acidity (\%) of Balady mandarin during cold storage in 2015 and 2016 seasons

\begin{tabular}{|c|c|c|c|c|c|c|c|c|c|c|}
\hline \multirow{3}{*}{ Treatments } & \multicolumn{5}{|c|}{2015 season } & \multicolumn{5}{|c|}{2016 season } \\
\hline & \multicolumn{10}{|c|}{ Storage periods in days at $2 \pm 1^{0} \mathrm{C}$} \\
\hline & $\mathbf{0}$ & 15 & 30 & 45 & Mean & $\mathbf{0}$ & 15 & 30 & 45 & Mean \\
\hline Control & $1.06 \mathrm{~d}$ & $0.97 \mathrm{~d}$ & $0.92 \mathrm{~d}$ & $0.90 \mathrm{c}$ & $0.97 \mathrm{~d}$ & $1.07 \mathrm{c}$ & $0.95 \mathrm{c}$ & $0.89 \mathrm{~d}$ & $0.90 \mathrm{c}$ & $0.96 \mathrm{~d}$ \\
\hline Kaolin $1 \%$ & $1.10 \mathrm{c}$ & $1.05 \mathrm{c}$ & $0.98 \mathrm{c}$ & $0.92 \mathrm{~b}$ & $1.01 \mathrm{c}$ & $1.11 \mathrm{~b}$ & $1.08 \mathrm{~b}$ & $1.00 \mathrm{c}$ & $0.92 \mathrm{bc}$ & $1.04 \mathrm{bc}$ \\
\hline Kaolin $2 \%$ & $1.12 \mathrm{~b}$ & $1.08 \mathrm{~b}$ & $1.02 \mathrm{~b}$ & $0.98 \mathrm{a}$ & $1.05 \mathrm{~b}$ & $1.12 \mathrm{ab}$ & $1.10 \mathrm{a}$ & $1.04 \mathrm{~b}$ & $0.94 \mathrm{a}$ & $1.03 \mathrm{c}$ \\
\hline Kaolin $3 \%$ & $1.12 \mathrm{~b}$ & $1.09 \mathrm{ab}$ & $1.04 \mathrm{ab}$ & $0.99 \mathrm{a}$ & $1.06 \mathrm{ab}$ & $1.13 \mathrm{ab}$ & $1.10 \mathrm{a}$ & $1.05 \mathrm{ab}$ & $0.96 \mathrm{a}$ & $1.05 \mathrm{ab}$ \\
\hline Kaolin $4 \%$ & $1.14 \mathrm{a}$ & $1.10 \mathrm{a}$ & $1.06 \mathrm{a}$ & $0.99 \mathrm{a}$ & $1.07 \mathrm{a}$ & $1.15 \mathrm{a}$ & $1.10 \mathrm{a}$ & $1.06 \mathrm{a}$ & $0.97 \mathrm{a}$ & $1.07 \mathrm{a}$ \\
\hline \multirow[t]{2}{*}{ Mean } & 1.10 & 1.05 & 1.00 & 0.95 & & 1.11 & 1.06 & 1.00 & 0.93 & \\
\hline & \multicolumn{10}{|c|}{ Storage periods in days at $6 \pm 1^{0} \mathrm{C}$} \\
\hline Control & $1.06 \mathrm{~d}$ & $0.97 \mathrm{e}$ & $0.95 \mathrm{~d}$ & $0.88 \mathrm{c}$ & $0.96 \mathrm{~d}$ & $1.07 \mathrm{c}$ & $0.95 \mathrm{c}$ & $0.91 \mathrm{a}$ & $0.83 b$ & $0.92 \mathrm{c}$ \\
\hline Kaolin $1 \%$ & $1.10 \mathrm{c}$ & $0.98 \mathrm{~d}$ & $0.96 \mathrm{c}$ & $0.92 \mathrm{~b}$ & $0.99 \mathrm{c}$ & $1.11 \mathrm{~b}$ & $0.97 \mathrm{bc}$ & $0.94 \mathrm{a}$ & $.0 .88 b$ & $0.97 \mathrm{bc}$ \\
\hline Kaolin $2 \%$ & $1.12 \mathrm{~b}$ & $1.00 \mathrm{c}$ & $0.98 \mathrm{~b}$ & $0.95 \mathrm{a}$ & $1.01 \mathrm{~b}$ & $1.12 \mathrm{ab}$ & $0.99 \mathrm{ab}$ & $0.96 \mathrm{a}$ & $0.95 \mathrm{ab}$ & $1.00 \mathrm{ab}$ \\
\hline Kaolin $3 \%$ & $1.12 \mathrm{~b}$ & $1.03 \mathrm{~b}$ & $0.98 b$ & $0.96 \mathrm{a}$ & $1.02 \mathrm{ab}$ & $1.13 \mathrm{ab}$ & $1.05 \mathrm{a}$ & $0.97 \mathrm{a}$ & $0.95 \mathrm{ab}$ & $1.03 \mathrm{a}$ \\
\hline Kaolin $4 \%$ & $1.14 \mathrm{a}$ & $1.04 \mathrm{a}$ & $0.99 \mathrm{a}$ & $0.96 \mathrm{a}$ & $1.03 \mathrm{a}$ & $1.15 \mathrm{a}$ & $1.05 \mathrm{a}$ & $0.98 \mathrm{a}$ & $0.96 \mathrm{a}$ & $1.03 \mathrm{a}$ \\
\hline Mean & 1.10 & 1.00 & 0.97 & 0.93 & & 1.11 & 1.00 & 0.95 & 0.91 & \\
\hline
\end{tabular}

Means followed by different letter are significantly different within columns by Duncan's multiple range test, $\mathrm{P} \leq .05$. highest values of V.C content at harvest and during storage period compared the other concentrations in the two seasons. Vit. $\mathrm{C}$ content in Balady mandarin fruits stored at two different temperatures, $2 \pm 1^{0} \mathrm{C}$ and $6 \pm 1{ }^{\circ} \mathrm{C}$ decreased gradually with the progress of storage period and with the increase of storage temperature. Moreover, fruits of trees treated with kaolin foliar application had significantly higher value of V.C compared to the control during storage period in both seasons. At both storage temperatures, the highest ascorbic acid content was observed at $4 \%$ kaolin treatment followed by $3 \%$ kaolin treatment, whereas, control treatment had the lowest value of ascorbic acid compared to other treatments.

So, it can be concluded that, kaolin foliar application at 3 and $4 \%$ are most effective in preventing V.C loss from fruits during storage period, this may be due to its effect in reducing fruit decay, dehydration and delay the decline in fruit quality. The same trend was also observed in the previous study by Ali and Elhamahmy (2015) who reported that, Washington navel orange fruits, sprayed with $2.5 \%$ kaolin had the maximum content of ascorbic acid at the end of storage period.

\section{CONCLUSION}

In light of this study it can be concluded that, preharvest kaolin foliar application in summer months specially with $4 \%$ which the was most effective treatment for increasing yield, reduce fruit disorders and enhancing fruit quality at harvest time and also, maintaining fruit quality and extending storability of Balady mandarin fruits during cold storage at $(2 \pm 1$ or $6 \pm 1^{0} \mathrm{C}$ ) which exhibited by the least weight loss, decay and chilling injuries, highest fruit firmness, titratable acidity, soluble solids and ascorbic acid contents . 
Table 9. Effect of kaolin foliar sprays on SSC/acid ratio of Balady mandarin during cold storage in 2015 and 2016 seasons

\begin{tabular}{|c|c|c|c|c|c|c|c|c|c|c|}
\hline \multirow{3}{*}{ Treatments } & \multicolumn{5}{|c|}{2015 season } & \multicolumn{5}{|c|}{2016 season } \\
\hline & \multicolumn{10}{|c|}{ Storage periods in days at $2 \pm 1^{0} \mathrm{C}$} \\
\hline & $\mathbf{0}$ & 15 & 30 & 45 & Mean & $\mathbf{0}$ & 15 & 30 & 45 & Mean \\
\hline Control & $10.64 \mathrm{a}$ & $11.47 \mathrm{a}$ & $11.96 \mathrm{a}$ & $12.25 \mathrm{a}$ & $11.58 \mathrm{a}$ & $10.75 \mathrm{a}$ & $11.93 \mathrm{a}$ & $12.51 \mathrm{a}$ & $12.14 \mathrm{c}$ & $11.83 \mathrm{a}$ \\
\hline Kaolin $1 \%$ & $10.71 \mathrm{a}$ & $11.23 \mathrm{a}$ & $11.90 \mathrm{a}$ & $12.51 \mathrm{a}$ & $11.59 \mathrm{a}$ & $10.94 \mathrm{a}$ & $11.11 \mathrm{~b}$ & $11.86 \mathrm{~b}$ & $12.61 \mathrm{a}$ & $11.63 \mathrm{ab}$ \\
\hline Kaolin $2 \%$ & $11.00 \mathrm{a}$ & $11.04 \mathrm{a}$ & $11.70 \mathrm{ab}$ & $12.00 \mathrm{a}$ & $11.44 \mathrm{a}$ & $10.99 \mathrm{a}$ & $10.96 \mathrm{~b}$ & $11.54 \mathrm{~b}$ & $12.55 \mathrm{ab}$ & $11.51 \mathrm{~b}$ \\
\hline Kaolin $3 \%$ & $11.08 \mathrm{a}$ & $11.01 \mathrm{a}$ & $11.47 \mathrm{~b}$ & $11.94 \mathrm{a}$ & $11.38 \mathrm{a}$ & $11.06 \mathrm{a}$ & $11.15 \mathrm{~b}$ & $11.55 \mathrm{~b}$ & $12.43 \mathrm{bc}$ & $11.55 \mathrm{~b}$ \\
\hline Kaolin $4 \%$ & $10.97 \mathrm{a}$ & $11.03 \mathrm{a}$ & $11.32 \mathrm{~b}$ & $12.01 \mathrm{a}$ & $11.33 \mathrm{a}$ & $10.92 \mathrm{a}$ & $11.30 \mathrm{~b}$ & $11.57 \mathrm{~b}$ & $12.51 \mathrm{ab}$ & $11.58 \mathrm{~b}$ \\
\hline \multirow[t]{2}{*}{ Mean } & 10.88 & 11.16 & 11.67 & 12.14 & & 10.93 & 11.29 & 11.81 & 12.45 & \\
\hline & \multicolumn{10}{|c|}{ Storage periods in days at $6 \pm 1^{0} \mathrm{C}$} \\
\hline Control & $10.64 \mathrm{a}$ & $11.13 \mathrm{~d}$ & $11.15 \mathrm{~b}$ & $11.56 \mathrm{a}$ & $11.12 \mathrm{c}$ & $10.75 \mathrm{a}$ & $12.12 b$ & $12.15 \mathrm{a}$ & $12.93 \mathrm{a}$ & $11.99 \mathrm{a}$ \\
\hline Kaolin $1 \%$ & $10.71 \mathrm{a}$ & $11.90 \mathrm{ab}$ & $11.86 \mathrm{a}$ & $12.10 \mathrm{a}$ & $11.64 \mathrm{ab}$ & $10.94 \mathrm{a}$ & $12.53 \mathrm{a}$ & $12.48 \mathrm{a}$ & $12.88 \mathrm{a}$ & $12.21 \mathrm{a}$ \\
\hline Kaolin $2 \%$ & $11.00 \mathrm{a}$ & $12.06 \mathrm{a}$ & $12.07 \mathrm{a}$ & $12.06 \mathrm{a}$ & $11.80 \mathrm{a}$ & $10.99 \mathrm{a}$ & $12.42 \mathrm{a}$ & $12.71 \mathrm{a}$ & $12.17 \mathrm{~b}$ & $12.07 \mathrm{a}$ \\
\hline Kaolin $3 \%$ & $11.08 \mathrm{a}$ & $11.51 \mathrm{c}$ & $11.95 \mathrm{a}$ & $11.98 \mathrm{a}$ & $11.63 \mathrm{~b}$ & $11.06 \mathrm{a}$ & $11.90 \mathrm{~b}$ & $12.43 \mathrm{a}$ & $12.27 b$ & $11.92 \mathrm{a}$ \\
\hline Kaolin $4 \%$ & $10.97 \mathrm{a}$ & $11.72 \mathrm{bc}$ & $11.97 \mathrm{a}$ & $12.08 \mathrm{a}$ & $11.69 \mathrm{ab}$ & $10.92 \mathrm{a}$ & $11.96 \mathrm{~b}$ & $12.38 \mathrm{a}$ & $12.35 \mathrm{ab}$ & $11.87 \mathrm{a}$ \\
\hline Mean & 10.88 & 11.66 & 11.80 & 11.95 & & 10.93 & 12.19 & 12.42 & 12.52 & \\
\hline
\end{tabular}

Means followed by different letter are significantly different within columns by Duncan's multiple range test, $\mathrm{P} \leq .05$.

Table 10. Effect of kaolin foliar sprays on fruit vitamin $\mathrm{C} \mathrm{mg} / 100 \mathrm{ml}$ juice of Balady mandarin during cold storage in 2015 and 2016 seasons

\begin{tabular}{|c|c|c|c|c|c|c|c|c|c|c|}
\hline \multirow{3}{*}{ Treatments } & \multicolumn{5}{|c|}{2015 season } & \multicolumn{5}{|c|}{2016 season } \\
\hline & \multicolumn{10}{|c|}{ Storage periods in days at $2 \pm 1^{\circ} \mathrm{C}$} \\
\hline & $\mathbf{0}$ & 15 & 30 & 45 & Mean & $\mathbf{0}$ & 15 & 30 & 45 & Mean \\
\hline Control & $39.20 \mathrm{c}$ & $34.19 \mathrm{e}$ & $33.51 \mathrm{e}$ & $32.09 \mathrm{e}$ & $34.75 \mathrm{~d}$ & $40.30 \mathrm{~d}$ & $34.33 \mathrm{e}$ & $33.86 \mathrm{c}$ & $32.99 \mathrm{e}$ & $35.37 \mathrm{e}$ \\
\hline Kaolin $1 \%$ & $43.80 \mathrm{~b}$ & $37.74 \mathrm{~d}$ & $36.94 \mathrm{~d}$ & $36.70 \mathrm{~d}$ & $38.80 \mathrm{c}$ & $44.80 \mathrm{c}$ & $37.79 \mathrm{~d}$ & $36.96 \mathrm{bc}$ & $37.11 \mathrm{~d}$ & $39.17 \mathrm{~d}$ \\
\hline Kaolin $2 \%$ & $43.70 \mathrm{~b}$ & $40.00 \mathrm{c}$ & $39.05 \mathrm{c}$ & $38.40 \mathrm{c}$ & $40.29 \mathrm{c}$ & $45.50 \mathrm{~b}$ & $39.97 \mathrm{c}$ & $39.76 \mathrm{ab}$ & $39.12 \mathrm{c}$ & $41.09 \mathrm{c}$ \\
\hline Kaolin $3 \%$ & $45.50 \mathrm{ab}$ & $43.38 \mathrm{~b}$ & $41.52 \mathrm{~b}$ & $41.37 \mathrm{~b}$ & $42.94 \mathrm{~b}$ & $45.80 \mathrm{~b}$ & $43.08 \mathrm{~b}$ & $42.08 \mathrm{a}$ & $41.21 \mathrm{~b}$ & $43.24 \mathrm{~b}$ \\
\hline Kaolin $4 \%$ & $46.50 \mathrm{a}$ & $44.85 \mathrm{a}$ & $44.05 \mathrm{a}$ & $43.39 \mathrm{a}$ & $44.70 \mathrm{a}$ & $46.60 \mathrm{a}$ & $44.65 \mathrm{a}$ & $44.06 \mathrm{a}$ & $43.77 \mathrm{a}$ & $44.57 \mathrm{a}$ \\
\hline \multirow[t]{2}{*}{ Mean } & 43.74 & 40.03 & 39.01 & 38.39 & & 44.60 & 39.96 & 39.34 & 38.84 & \\
\hline & \multicolumn{10}{|c|}{ Storage periods in days at $6 \pm 1^{0} \mathrm{C}$} \\
\hline Control & $39.20 \mathrm{c}$ & $34.10 \mathrm{e}$ & $32.48 \mathrm{e}$ & $29.81 \mathrm{~d}$ & $33.90 \mathrm{~d}$ & $40.30 \mathrm{~d}$ & $34.31 \mathrm{e}$ & $33.00 \mathrm{~d}$ & $30.71 \mathrm{~d}$ & $33.20 \mathrm{e}$ \\
\hline Kaolin $1 \%$ & $43.80 \mathrm{~b}$ & $36.79 \mathrm{~d}$ & $35.94 \mathrm{~d}$ & $33.19 \mathrm{c}$ & $37.43 \mathrm{c}$ & $44.80 \mathrm{c}$ & $37.46 \mathrm{~d}$ & $36.42 \mathrm{c}$ & $34.00 \mathrm{c}$ & $36.72 \mathrm{~d}$ \\
\hline Kaolin $2 \%$ & $43.70 \mathrm{~b}$ & $39.33 c$ & $36.94 \mathrm{c}$ & $33.36 \mathrm{c}$ & $38.33 \mathrm{c}$ & $45.50 \mathrm{~b}$ & $39.48 \mathrm{c}$ & $37.77 \mathrm{c}$ & $34.86 \mathrm{c}$ & $38.39 \mathrm{c}$ \\
\hline Kaolin $3 \%$ & $45.50 \mathrm{ab}$ & $41.06 \mathrm{~b}$ & $39.93 \mathrm{~b}$ & $35.72 b$ & $40.55 \mathrm{~b}$ & $45.80 \mathrm{~b}$ & $42.60 \mathrm{~b}$ & $40.96 \mathrm{~b}$ & $38.39 \mathrm{~b}$ & $41.32 \mathrm{~b}$ \\
\hline Kaolin $4 \%$ & $46.50 \mathrm{a}$ & $44.00 \mathrm{a}$ & $42.78 \mathrm{a}$ & $40.09 \mathrm{a}$ & $43.34 \mathrm{a}$ & $46.60 \mathrm{a}$ & $44.75 \mathrm{a}$ & $43.33 \mathrm{a}$ & $40.38 \mathrm{a}$ & $43.49 \mathrm{a}$ \\
\hline Mean & 43.74 & 38.45 & 37.61 & 34.43 & & 44.60 & 39.72 & 38.29 & 40.15 & \\
\hline
\end{tabular}

Means followed by different letter are significantly different within columns by Duncan's multiple range test, $\mathrm{P} \leq .05$.

\section{REFERENCES}

Abd-Allah, A. S., E. Abd El-Razek and M. M. Saleh. 2013. Effect of sun-block materials on preventing sunburn injury of Keitt mango fruits. J. Appl. Sci. Res., 9 (1) : $567-571$.

Abdel Ghani, N. A., M. A. Galal, M. E. El-Sayed, S. M. ElMarsafawy and M. A. Omran. 2013. Effect of spraying kaolin and calcium carbonate on the productivity of Aggezi and Picual olive cvs. J. Plant Production, Mansoura Univ., 4(7) : 1035 - 1050.

Ahmad, S., Z. Singh, A. S. Khan and Z. Iqbal. 2013. Preharvest application of salicylic acid maintain the rind textural properties and reduce fruit rot and chilling injury of Sweet orange during cold storage. Pak. J. Agri. Sci., 50 (4) : $559-569$.

Ali, M. S. and M. A. Elhamahmy. 2015. Impact of kaolin particles film coating and UV-C treatments on storability and quality of Washington navel orange during long period cold storage. Zagazig J. Agric. Res., 42 (5) : 1081 1099.

A.O.A.C. 1990. Association of official analytical chemists. Official Methods of Analysis. 15th Ed. Washington D. C., USA.

Aquino, S. D., A. Cocco, S. Ortu and M. Schirra. 2011. Effects of kaolin-based particle film to control Ceratitiscapitata (Diptera : Tephritidae) infestations and postharvest decay in citrus and stone fruit. Crop Protection. 30 : 1079 - 1086.

Argiriou, S. and G. D. Nanos. 2010. Preharvest AVG and kaolin and postharvest 1-MCP application effects on advanced maturity peach quality and storage. Acta Hort., 877: $311-316$.

Azizi, A., H. Hokmabadi, S. Piri and V. Rabie. 2013. Effect of kaolin application on water stress in pistachio cv. Ohadi. 
Journal of Nuts. 4 (4) : $9-14$.

Braham, M., E. Pasqualini and N. Ncira. 2007. Efficacy of kaolin, spinosad and malathion against Ceratitiscapitata in Citrus orchards. Bulletin of Insectology. 60 (1) : $39-47$.

Burton, W. G. 1985. Postharvest physiology of food crops. Longman Scientific and Technical. New York. USA.

Chabbal, M. D., A. B. Piccoli., G. C. Martinez., M. M. Avanza., S. M. Mazza and V. A. Rodriguez. 2014. Kaolin applications to control sunburn in 'Okitsu' mandarin. Cultivos Tropicalesm. 35 (1) : $50-56$.

Colavita, G. M., V. Blackhall and S. Valdez. 2011. Effect of kaolin particle films on the temperature and solar injury of pear fruits. Acta Hort. 909 : $609-615$.

Du Plooy, G. W. 2006. Aspects of mango (Mangifera indica L.) fruit rind morphology and chemistry and their implication for postharvest quality. Ph. D. Thesis. Faculty of Natural and Agricultural Sciences, University of Pretoria, Pretoria, South Africa.

Duncan, D. B. 1955. Multiple range and multiple F. test. Biometries. $11: 1-42$.

El-Khawaga, A. S. and A. E .Mansour. 2014. Enhancing the efficiency of irrigation water use by using some antitranspirants in Wonderful pomegranate orchards. Middle East J. Agric. Res., 3 (3) : $694-700$.

Ennab, H. A., S. A. El-Sayed and M. M. Abo El-Enin. 2017. Effect of kaolin applications on fruit sunburn, yield and fruit quality of Balady mandarin (Citrus reticulata, Blanco). Menoufia J. Plant Prod., 2 : 129 - 138.

Ergun, M. 2012. Postharvest quality of 'Galaxy' apple fruit in response to kaolin based particle film application. J. Agric. Sci. Technol. 14 : $599-607$.

Glenn, D. M. 2009. Particle film mechanisms of action that reduce the effect of environmental stress in Empire apple. J. Amer. Soc. Hort. Sci., 134 (3) :314 - 321.

Glenn, D. M. and G. J. Puterka. 2005. Particle films : A new technology for agriculture. Hort. Rev., (Amer. Soc. Hort. Sci.) $31: 1-44$.

Glenn, D. M., E. Prado, A. Erez, G. M. Ferson and G. J. Puterka. 2002. A reflective processed kaolin particle film affects fruit temperature, radiation reflection and solar injury in apple. J. Amer. Soc. Hort. Sci., 126 (2) :188193.

Hegazi, A., N. R. Samra. E. E. El-Baz. B. M. Khalil and M. S.
Gawish. 2014. Improving fruit quality of Manfalouty and Wonderful pomegranate by using bagging and some spray treatments with gibberellic acid, calcium chloride and kaolin. J . Plant Production, Mansoura Univ., 5 (5) : 779 792.

Kumer, R. and V. Kumer. 2016. Physiological disorders in perennial woody tropical and subtropical fruit crops : A review Indian J. Agric. Sci., 86 (6) :703 - 17.

Mditshwa, A., J. P. Bower, I. Bertling, N. Mathaba and S. Z. Tesfay. 2013. The potential of postharvest silicon dips to regulate phenolics in citrus peel as a method to mitigate chilling injury in lemons. African Journal of Biotechnology. 12 (13) : 1482 - 1489.

Minessy, F.A., T.A. Nasr and M.Y. El-Shurafa. 1969. Citrus fruit temperature in relation to sunburn. Proceceedings of the Conference on Tropical and Subtropical fruits. London, England. pp. $245-252$.

Obaje, S.O. J. I. Omada and U.A. Dambatta. 2013. Clays and their industrial applications: Synoptic review. Inter. J. Sci. \& Tech., 3 (5) : $264-270$.

Palitha, W.J., M. Magdalena and J. Rogers. 2010. The effect of maturity, sunburn and application of sunscreen on the internal and external qualities of pomegranate fruit grown in Australia. Scientia Hort., 124: 57 - 61.

Schrader, L.E. 2011. Scientific basis of a unique formulation for reducing sunburn of fruits. HortScience. 46 (1) : $6-$ 11.

Schrader, L.E., J. Zhang, J. Sun, J. Xu, D. C. Elfving and C. Kahn. 2009. Postharvest changes in internal fruit quality in apples with sunburn browning. J. Amer. Soc. Hort. Sci., 34 (1): $148-155$

Vatandoost, S., G.H. Davarynejad and A. Tehranifar . 2014. Would kaolin particle film avoid sunburn in Ardestani pomegranate. Advances in Environmental Biology. 8 (12): 607-610.

Verreynne, S. and S.V. Merwe. 2011. Sunburn reduction on 'Miho Wase' Satsuma mandarin. South Africa Fruit Journal. 10 (2) : $52-55$.

Weerakkody, P., J. Jobling, M.M. Infante and G. Rogers. 2010. The effect of maturity, sunburn and the application of sunscreens on the internal and external qualities of pomegranate fruit grown in Australia. Scientia Horticulturae. 124:57 - 61. 


\section{الملخص العربي}

\section{تأثير الرش بالكاولين على جودة وطول القترة التخزينية لثمار اليوسفى البلدى}

$$
\text { على السيد زغلول، حسن أبو الفتوح عناب، مرفت عبد المجيد الثيمى }
$$

هذا بالإضـافة إلى أن الــرش بالكــاولين بتركيـز ع ؛ كانت المعاملة الأكثر تأثير ا في الحفاظ على جـــودة ثــــار

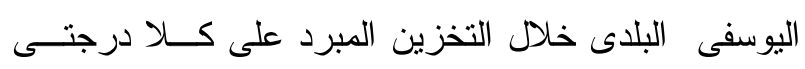

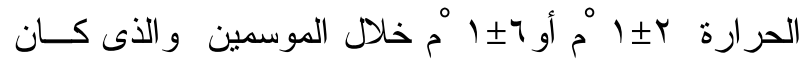
و اضحا معنويا في خفض النسبة المئوية لفقد الوزن ونسـبة مبة التلف و أضر ار البــرودة أيضـــا. كمـــا ســـلت هاتــان المعاملاتان من الرش بالكاولين (ع أوب \%) القيم الأعلى

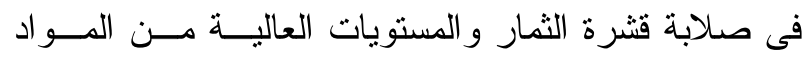
الصلبة الذائبة الكلية و الحموضة و المحتوى من فيتامين جل ج

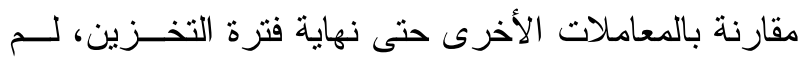
تكن هنالك تأثثر ات معنوية في نسبة المو اد الصــلبة الذائبــة الكلية|الحموضة.

و على ذلك، يعتبر الرش بالكاولين بتركيـز ع ٪ ث ثـلاث مرات خلال أثهر الصيف، كانت الأكثر تأثير ا فى خفض نسبة أضرار الثمار قبل الحصاد وتحسين المحصول وجودة الثمار عند الحصاد و الحفاظ على الجودة وزيــادة القـدرة التخزينية لثمار اليوسفى البلدى عنة في المعاملات الأخرى أو الكنترول خلال التخزين المبرد على كلا درجتى الحرارة
أجريث هذه الدراسة في مزرعة خاصة فى مركز مطوبس، محافظة كفر الشيخ، مصر خلال موسمى ع ا . r-

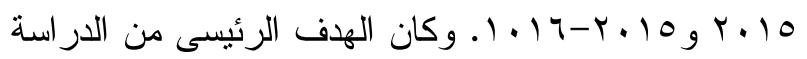
هو تقييم تأثير رش الكاولين على أضرار الثمار و المحصول وجودة و القدرة التخزينية لثمار اليوسفي البلدي. ثم رش أشجار اليوسفى البلدى بخمس ثركيزات من الكاولين هم) • و او ب وب و ع \% ) ثلاث مرات خلال أشهر الصيف (يونيو ويوليووأغسطس). جمعت عينات الثمار لتقدير صفاتها. خزنت عينات الثمار المتبقية على درجنى حرارة بـأم أو أو

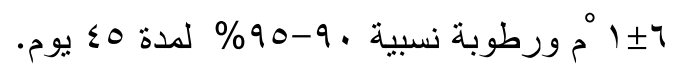
أظهرت النتائج أن الرش بالكــاولين أدى إلــى زيـــادة المحصول (عدد ووزن وكجم ا شجرة) وخفض من نســبة أضرار الثمار وحسن من جودة الثمار حيث سجلت زيــادة فى صلابة قشرة الثمار ومستويات الحموضة ونسبة المواد الصلبة الذائبة الكلية و المحتوى من فيتامين ج عند الحصاد.

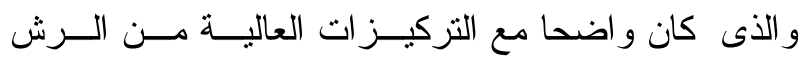
بالكاولين (ع\%) وكانت الإختلافــات معنويـــة بالمقارنــة بالمعاملات الأخرى و الكنترول. 